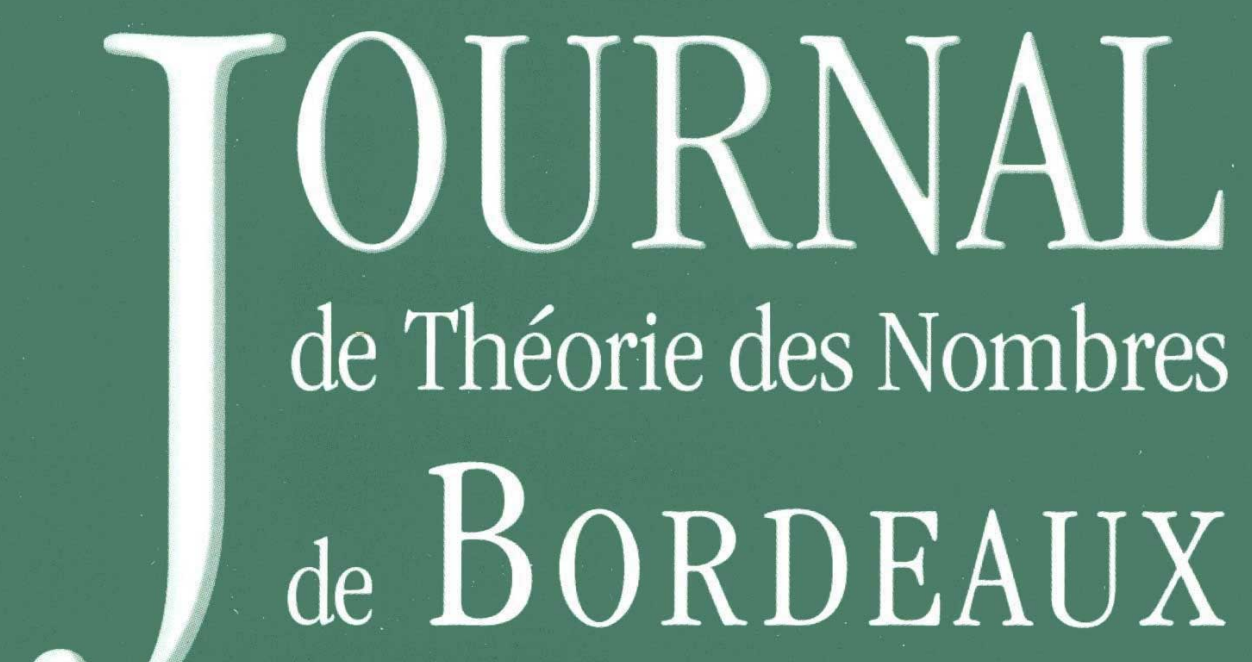

anciennement Séminaire de Théorie des Nombres de Bordeaux

Daniel VALLIÈRES

\title{
Connected abelian complex Lie groups and number fields
}

Tome 24, no 1 (2012), p. 201-229.

<http://jtnb.cedram.org/item?id=JTNB_2012__24_1_201_0>

(C) Société Arithmétique de Bordeaux, 2012, tous droits réservés.

L'accès aux articles de la revue «Journal de Théorie des Nombres de Bordeaux » (http://jtnb.cedram.org/), implique l'accord avec les conditions générales d'utilisation (http://jtnb.cedram. org/legal/). Toute reproduction en tout ou partie cet article sous quelque forme que ce soit pour tout usage autre que l'utilisation à fin strictement personnelle du copiste est constitutive d'une infraction pénale. Toute copie ou impression de ce fichier doit contenir la présente mention de copyright.

\section{cedram}




\title{
Connected abelian complex Lie groups and number fields
}

\author{
par DANIEL VALLIÈRES
}

\begin{abstract}
RÉsumÉ. Dans cet article, nous expliquons une façon d'associer à tout corps de nombres certains groupes de Lie complexes et connexes. Nous étudions en particulier le cas des corps de nombres de degré 3 sur $\mathbb{Q}$ qui ne sont pas totalement réels et expliquons le lien entre ceux-ci et les groupes de Cousin ("groupes toroidaux") de dimension complexe 2 et de rang 3 .
\end{abstract}

ABSTRACT. In this note we explain a way to associate to any number field some connected complex abelian Lie groups. Further, we study the case of non-totally real cubic number fields, and we see that they are intimately related with the Cousin groups (toroidal groups) of complex dimension 2 and rank 3.

\section{Contents}

1. Introduction

2. Connected abelian complex Lie groups

2.1. Endomorphisms of connected abelian complex Lie groups

2.2. Matrix notation

3. Non-totally real cubic number fields

3.1. The Remmert-Morimoto decomposition

3.2. The ring of endomorphisms

3.3. The action of the class group

\section{Introduction}

The theory of complex multiplication started with Abel and was developed later on by Kronecker and Weber. Hilbert and his students Takagi, Fueter and Hecke studied the theory further followed by Hasse and his student Deuring who developed another approach which was more algebraic.

\footnotetext{
Manuscrit reçu le 9 septembre 2010, révisé le 27 janvier 2011.

Research supported by the NSERC.
} 
They made fundamental use of elliptic curves and their reduction modulo various primes. Shimura, Taniyama and Weil extended this theory to abelian varieties. It has always been a rich theory involving several different areas of mathematics.

Kronecker conjectured that every abelian extension of a quadratic imaginary number field $K$ can be generated by special values of the $j$-function evaluated at some points in $K$ which are also in the upper-half plane. It turns out that this conjecture was false as noticed by Fueter. One has to use special values of some modified elliptic functions, the so-called Weber functions, in order to get $K^{a b}$. We remark here that if the class number of $K$ is one, then one can just use the values of a single elliptic function (associated with a single lattice $\Gamma$ ) evaluated at torsion points of $\mathbb{C} / \Gamma$ in order to get $K^{a b}$, a fact which could be considered as the analogue of what is going on for the class number one number field $\mathbb{Q}$ and the periodic function $z \mapsto \exp 2 \pi i z$. This is in fact what Takagi did in his Ph.D. thesis under Hilbert's supervision in the special case of $\mathbb{Q}(i)$ using the lemniscate function.

Another student of Hilbert, Hecke, tried to generalize the theory for quadratic imaginary number fields to other number fields, the so-called $C M$-fields of degree 4 over $\mathbb{Q}$. He considered his attempt as a failure. Nevertheless, after the Bordin's prize memoir of Leftschetz and the work of Weil on the Riemann hypothesis in function fields, the theory of abelian varieties gave a new perspective on the subject. The theory of complex multiplication of abelian varieties was developed by Shimura, Taniyama and Weil. To any pair $(K, \Phi)$ where $K$ is a $C M$-field and $\Phi$ a $C M$-type, there is another $C M$-field associated to it called the reflex field. If $K$ is an abelian extension of $\mathbb{Q}$ and $\Phi$ is not lifted from any smaller $C M$-field, it turns out that its reflex field coincides with $K$, and this is why the reflex field does not show up in the classical theory of complex multiplication of elliptic curves, since a quadratic imaginary number field clearly does not contain any smaller $C M$-field (there are other instances when the $C M$-field $K$ and its reflex field coincide). Moreover, one first uses special values of Siegel modular functions (or Hilbert modular functions) in order to get unramified abelian extensions of its reflex field (even though it is known that in general, one does not get the full Hilbert class field), and then one uses some modified abelian functions in order to generate some abelian extensions again of the reflex field. Furthermore, this theory gives an explicit formula for the action of the Artin symbol on these algebraic points. In the classical case of elliptic curves, the classical $j$-function is such a Siegel modular function and the modified elliptic functions are the so-called Weber functions. We refer to [7] and the expanded [8] for this theory and also to [6] for an emphasis on the elliptic curve case. 
We see that this theory is a mixture of the following ingredients.

- The complex points of an abelian variety defined over $\mathbb{C}$ form a complex torus, i.e. a compact complex abelian Lie group. This can be viewed as the analytico-geometric point of view of the question.

- An abelian variety is a projective algebraic group, and this is the point of view from algebraic geometry.

- The abelian functions on a complex torus (and their associated theta functions) allow one to embed it in a projective space as an algebraic group under a certain condition, namely the existence of a polarization.

- The automorphic functions which can be viewed in our case as functions on some moduli spaces of abelian varieties with extra structure.

The following paper is just concerned with the analytico-geometric point of view, i.e. with some complex abelian Lie groups.

Let $p(X)$ be a polynomial with rational coefficients which is irreducible, and let $K=\mathbb{Q}[X] /(p(X))$ be the corresponding number field, which we do not view as embedded in $\mathbb{C}$. Suppose also that we are given a complete set of representatives modulo complex conjugation for the embeddings of $K$, say

$$
\Phi=\left\{\sigma_{1}, \ldots, \sigma_{r_{1}}, \sigma_{r_{1}+1}, \ldots, \sigma_{r_{1}+r_{2}}\right\}
$$

where the first $r_{1}$ embeddings are the real ones. Out of these data, one gets a Minkowski map

$$
\mu_{\Phi}: K \rightarrow \mathbb{C}^{r_{1}+r_{2}},
$$

defined as usual by the formula $\lambda \mapsto\left(\sigma_{1}(\lambda), \ldots, \sigma_{r_{1}+r_{2}}(\lambda)\right)$. If $\mathfrak{m}$ is any module of $K$, that is a free abelian group contained in $K$ of $\operatorname{rank}[K: \mathbb{Q}]$, then $\mu_{\Phi}(\mathfrak{m})$ is a lattice in $\mathbb{C}^{r_{1}+r_{2}}$ not necessarily of full rank (see Theorem 4). This gives a systematic way of associating to these data a connected abelian complex Lie group, namely $\mathbb{C}^{r_{1}+r_{2}} / \mu_{\Phi}(\mathfrak{m})$.

When $K=\mathbb{Q}$, and $\mathfrak{m}=\mathbb{Z}$, one gets $\mathbb{C} / \mathbb{Z}$ which is an example of a noncompact connected abelian complex Lie group, and as it is well-known the singular values

$$
\exp \left(2 \pi i \frac{m}{n}\right)
$$

of the exponential function $z \mapsto \exp (2 \pi i z)$, viewed as a function on $\mathbb{C} / \mathbb{Z}$, generate abelian extensions of $\mathbb{Q}$ and if fact all of them. Another way of saying this fact is that, the values obtained by evaluating the exponential function at the torsion points of $\mathbb{C} / \mathbb{Z}$ generate abelian extensions of $\mathbb{Q}$. This is the so-called Kronecker-Weber theorem and a proof can be found in Hilbert's Zahlbericht for instance.

When $K$ is a quadratic imaginary number field and $\mathfrak{m}$ is a fractional ideal of $K$ one gets the usual complex tori of complex dimension 1 admitting 
complex multiplication by the full ring of integers of $K$. Embedding them in a projective space gives a way to construct elliptic curves with complex multiplication by the full ring of integers $O_{K}$.

Even more generally, let $K$ be a $C M$-field (that is a totally imaginary number field which is a quadratic extension of a totally real number field as, for instance, the cyclotomic fields or the quadratic imaginary fields) of degree $2 n$ and suppose moreover that $\mathfrak{m}$ is a fractional ideal of $K$. Then $\mathbb{C}^{n} / \mu_{\Phi}(\mathfrak{m})$ is an example of a complex torus admitting a Riemann form. Therefore, according to the Riemann bilinear relations which are part of the classical theory of abelian functions as contained in [9] for instance, one concludes that there exists a non-degenerate abelian function on this torus, a phenomenon which is not always the case if $n \geq 2$. Similarly as in the imaginary quadratic number field case, one can use this fact in order to embed these complex tori in a projective space and this gives a way to construct abelian varieties with complex multiplication by the full ring of integers of $K$.

We point out that the known proofs of the theory of complex multiplication of abelian varieties make fundamental use of the GAGA principle. In other words, one makes use of the compactness of the complex torus especially for its application to explicit class field theory.

On the other hand, the case of $\mathbb{Q}$, even though it is a really special case, could excite the curiosity of someone and lead him to have a look at the non-compact complex Lie groups $\mathbb{C}^{r_{1}+r_{2}} / \mu_{\Phi}(\mathfrak{m})$ arising from some number fields which are not totally complex. After the quadratic imaginary number fields and the quartic $C M$-fields, the simplest fields which we could think of are the non-totally real cubic number fields. While thinking about this question, we discovered that some of the results presented here were already known to F. Gherardelli who wrote a paper in 1989 on this matter, but from a slightly different point of view, see [3]. Since we plan to study this subject further, we supply here possible proofs and in fact we just follow the classical line of thought in the case of complex tori.

We emphasize again that here, we are only concerned with the analyticogeometric side of the question, and we postpone the study of meromorphic functions on these connected abelian complex Lie groups to a further paper. We just remark that in the case of non-totally real cubic number fields, these functions are meromorphic functions of two complex variables having three $\mathbb{R}$-linearly independent periods and that these functions were first studied by Cousin in [2].

This paper is divided as follows. Section 2 recalls some properties of connected abelian complex Lie groups and especially the Remmert-Morimoto decomposition theorem. Section 2.1 is concerned with the endomorphisms of a connected abelian complex Lie group. The link with non-totally real 
cubic number fields starts in Section 3 where we compute the RemmertMorimoto decomposition of some connected abelian complex Lie groups arising from such number fields. Section 3.2 is concerned with the computation of the ring of endomorphisms of these complex Lie groups, and at last we explain an action of the class group on these complex Lie groups in Section 3.3. The main result of this paper is probably Corollary 7.

\section{Connected abelian complex Lie groups}

It is well-known that any connected abelian real analytic Lie group is isomorphic to one of the form $\mathbb{R}^{a} \times S^{m}$ where $S$ is the unit circle (here, $S^{m}$ is not the $m$ th-sphere in the $(m+1)$-dimensional euclidean space which is not even a group in general, but rather the cartesian product of $S$ with itself $m$ times). See for instance [4]. On the other hand, the structure theorem for connected abelian complex Lie groups is not as simple as in the real case. The purpose of this section is to explain and state this structure theorem (the Remmert-Morimoto decomposition, see Theorem 3).

In this section, except otherwise specified, morphisms (or isomorphisms) are always understood to be morphisms in the category of complex Lie groups (that is a morphism is a group homomorphism which is also $\mathbb{C}$ differentiable). First of all, we recall that a lattice $\Gamma$ in $\mathbb{C}^{n}$ is a closed discrete subgroup of $\mathbb{C}^{n}$. It is well-known that they are of the form

$$
\Gamma=\mathbb{Z} \cdot \gamma_{1}+\ldots+\mathbb{Z} \cdot \gamma_{t},
$$

where the $\gamma_{i}$ are $\mathbb{R}$-linearly independent, and $t \leq 2 n$. If $t=2 n$, we shall say that $\Gamma$ is a lattice of full rank.

Definition 1. Let $\Gamma$ be a lattice in $\mathbb{C}^{n}$. By a basis for $\Gamma$, we mean a set of $\mathbb{Z}$-generators $\gamma_{1}, \ldots, \gamma_{t}$ which are also $\mathbb{R}$-linearly independent.

Given a lattice $\Gamma$ in $\mathbb{C}^{n}$, the quotient $\mathbb{C}^{n} / \Gamma$ is our first example of a connected abelian complex Lie group. It turns out that all such groups are of this form:

Theorem 1. Let $G$ be a connected abelian complex Lie group of complex dimension $n$. There exists then a lattice $\Gamma$ in $\mathbb{C}^{n}$ such that

$$
G \simeq \mathbb{C}^{n} / \Gamma
$$

Proof. The proof is standard. One point of view consists of working with the universal covering group, whereas the other point of view uses the exponential map of Lie theory.

Definition 2. Let $G$ be a connected abelian complex Lie group. Any isomorphism

$$
\xi: G \rightarrow \mathbb{C}^{n} / \Gamma
$$

will be called a lattice representation of $G$. 
Notation. If $G$ is a connected abelian complex Lie group and $\xi: G \rightarrow \mathbb{C}^{n} / \Gamma$ is a lattice representation, then there is an isomorphism between $\operatorname{End}(G)$ and $\operatorname{End}\left(\mathbb{C}^{n} / \Gamma\right)$ given by

$$
\phi \mapsto \xi \circ \phi \circ \xi^{-1} .
$$

We shall denote this isomorphism by $\tilde{\xi}$.

It goes without saying that every time we make a definition about a connected abelian complex Lie group using a lattice representation, one has to check that this property does not depend on the choice of it. For instance, it makes sense to talk about the rank of a connected abelian complex Lie group (one could use Lemma 2 below in order to see that):

Definition 3. Let $G$ be a connected abelian complex Lie group and choose a lattice representation $G \simeq \mathbb{C}^{n} / \Gamma$. One defines the rank of $G$ to be

$$
\operatorname{rank}(G):=\operatorname{rank}_{\mathbb{Z}}(\Gamma) .
$$

Definition 4. A connected abelian complex Lie group $G$ having no other holomorphic functions then the constant ones will be called a Cousin group.

Remark. In the literature, a Cousin group is sometimes called a $(H, C)$ group or a toroidal group.

For example, any complex torus is a Cousin group, since there are no nonconstant holomorphic maps on a compact complex manifold. On the other hand, there are also non-compact connected abelian complex Lie groups which are Cousin. We will encounter such examples later on in connection with arithmetic.

So far, a connected complex abelian Lie group has two invariants. One is the complex dimension and the other one is the rank. We introduce another one.

Definition 5. Let $G$ be a connected abelian complex Lie group, and choose a lattice representation $G \simeq \mathbb{C}^{n} / \Gamma$. We define the complex rank of $G$ to be

$$
\operatorname{rank}_{\mathbb{C}}(G):=\operatorname{dim}_{\mathbb{C}}\left(\operatorname{Span}_{\mathbb{C}}(\Gamma)\right) \text {. }
$$

Remark. One can easily check that this notion is also well-defined.

Remark. We shall also write $\operatorname{rank}_{\mathbb{C}}(\Gamma)$ for $\operatorname{dim}_{\mathbb{C}}\left(\operatorname{Span}_{\mathbb{C}}(\Gamma)\right)$.

It is simple to see that if $\operatorname{rank}_{\mathbb{C}}(\mathrm{G})<n$ then $G$ is not Cousin, and since we clearly have

$$
\operatorname{rank}(G) \geq \operatorname{rank}_{\mathbb{C}}(\mathrm{G})
$$

we see that $G$ Cousin implies that $\operatorname{rank}(G) \geq n$. Here is a simple lemma whose proof is left to the reader. 
Lemma 1. Let $\Gamma \subseteq \mathbb{C}^{n}$ be a lattice of rank $n+m$ for some $m \geq 0$. Suppose also that $\operatorname{rank}_{\mathbb{C}}(\Gamma)=n$, then there exists a change of basis for which the lattice $\Gamma$ becomes

$$
\mathbb{Z}^{n} \oplus \Lambda
$$

where $\Lambda$ is another lattice of rank $m$. Moreover, the imaginary part of the lattice $\Lambda$, which we denote by $\operatorname{Im}(\Lambda)$, is of rank $m$ as well.

As an immediate corollary, we get:

Corollary 1. Let $G$ be a connected abelian complex Lie group of complex dimension $n$, and suppose

$$
\operatorname{rank}(G)=\operatorname{rank}_{\mathbb{C}}(G)=n
$$

then $G \simeq\left(\mathbb{C}^{\times}\right)^{n}$.

We already know that if $G$ is a Cousin group, then $\operatorname{rank}_{\mathbb{C}}(G)=n$. It follows moreover from this last corollary that necessarily $\operatorname{rank}(G)=n+m$ for some $m \geq 1$.

We now give a characterization of Cousin groups which is quite handy. We present the proof contained in the beautiful paper [1].

Theorem 2 (Irrationality condition). Let $\mathbb{C}^{n} / \Gamma$ be a connected abelian complex Lie group, and suppose that $\operatorname{rank}_{\mathbb{C}}(\Gamma)=n$. Then $\mathbb{C}^{n} / \Gamma$ is Cousin if and only if there does not exist any non-zero $L \in \operatorname{Hom}_{\mathbb{C}}\left(\mathbb{C}^{n}, \mathbb{C}\right)$ such that $L(\Gamma) \subseteq \mathbb{Z}$.

Proof. Because of Lemma 1 , we can suppose that $\Gamma=\mathbb{Z}^{n} \oplus \Lambda$ for some other lattice $\Lambda$. Let $f \in \operatorname{Hol}\left(\mathbb{C}^{n} / \Gamma\right)$, since in particular $f$ is $\mathbb{Z}^{n}$-periodic, $f$ has a Fourier expansion

$$
f(z)=\sum_{m \in \mathbb{Z}^{n}} a_{m} \exp \left(2 \pi i z m^{t}\right)
$$

converging everywhere on $\mathbb{C}^{n}$. The $\Lambda$-periodicity tells us that

$$
f(z+\lambda)=f(z) \text { for all } \lambda \in \Lambda,
$$

and in terms of Fourier series:

$$
\sum_{m \in \mathbb{Z}^{n}} a_{m} \exp \left(2 \pi i(z+\lambda) m^{t}\right)=\sum_{m \in \mathbb{Z}^{n}} a_{m} \exp \left(2 \pi i z m^{t}\right),
$$

for all $\lambda \in \Lambda$. This last equation implies then the following one

$$
\sum_{m \in \mathbb{Z}^{n}} a_{m} \exp \left(2 \pi i \lambda m^{t}\right) \exp \left(2 \pi i z m^{t}\right)=\sum_{m \in \mathbb{Z}^{n}} a_{m} \exp \left(2 \pi i z m^{t}\right),
$$

for all $\lambda \in \Lambda$. Since the Fourier coefficients are uniquely determined, we get

$$
a_{m}\left(\exp \left(2 \pi i \lambda m^{t}\right)-1\right)=0
$$


In other words, $a_{m}=0$ unless maybe $\lambda m^{t} \in \mathbb{Z}$ for all $\lambda \in \Lambda$. Consider then the set

$$
S=\left\{m \in \mathbb{Z}^{n} \mid \lambda m^{t} \in \mathbb{Z} \text { for all } \lambda \in \Lambda\right\} .
$$

We see that $\mathbb{C}^{n} / \Gamma$ is Cousin if and only if $S=\{0\}$.

We are just left to prove that $S=\{0\}$ if and only if there does not exist any non-zero $L \in \operatorname{Hom}_{\mathbb{C}}\left(\mathbb{C}^{n}, \mathbb{C}\right)$ such that $L(\Gamma) \subseteq \mathbb{Z}$, but this is clear because if $m \in S$ and $m \neq 0$ then the map $z \mapsto z m^{t}$ is such a $\mathbb{C}$-linear map. Conversely if such a $L$ exists and we express it in matrix notation using the canonical basis of $\mathbb{C}^{n}$, we see that it has to be of the form $z \mapsto z m^{t}$ for some non-zero $m \in \mathbb{C}^{n}$, but since $L\left(\mathbb{Z}^{n} \oplus \Lambda\right) \subseteq \mathbb{Z}$, we necessarily have $m \in \mathbb{Z}^{n}$.

We end this section with the statement of the structure theorem for connected complex abelian Lie groups.

Theorem 3 (Remmert-Morimoto decomposition). Any connected abelian complex Lie group $G$ is isomorphic to a group of the form

$$
\mathbb{C}^{a} \times\left(\mathbb{C}^{\times}\right)^{b} \times G_{0},
$$

where $G_{0}$ is a Cousin group. Moreover, the decomposition is unique meaning that if

then

$$
\mathbb{C}^{a} \times\left(\mathbb{C}^{\times}\right)^{b} \times G_{0} \simeq \mathbb{C}^{a^{\prime}} \times\left(\mathbb{C}^{\times}\right)^{b^{\prime}} \times G_{0}^{\prime},
$$

$$
a=a^{\prime}, b=b^{\prime}, \text { and } G_{0} \simeq G_{0}^{\prime} .
$$

Proof. The idea of the proof contained in [5] is to look at the unique closed connected complex normal subgroup $G_{0}$ such that $G / G_{0}$ is a Stein group and such that $G_{0}$ is a Cousin group. One then shows that $G \simeq G_{0} \times G / G_{0}$ and uses the fact that a Stein group is of the form $\mathbb{C}^{a} \times\left(\mathbb{C}^{\times}\right)^{b}$. The details of the proof can be found in [5].

2.1. Endomorphisms of connected abelian complex Lie groups. Let $G$ be a connected abelian complex Lie group. We shall denote by $\operatorname{End}(G)$ the ring of endomorphisms of $G$. One of the important uses of lattice representations is that it allows one to study the ring of endomorphisms of a connected abelian complex Lie group.

Lemma 2. Let $\phi: \mathbb{C}^{n} / \Gamma_{1} \rightarrow \mathbb{C}^{m} / \Gamma_{2}$ be a morphism of connected abelian complex Lie groups. There exists then a unique $\mathbb{C}$-linear map $L_{\phi}: \mathbb{C}^{n} \rightarrow \mathbb{C}^{m}$ such that $L_{\phi}\left(\Gamma_{1}\right) \subseteq \Gamma_{2}$ and such that the following diagram is commutative:

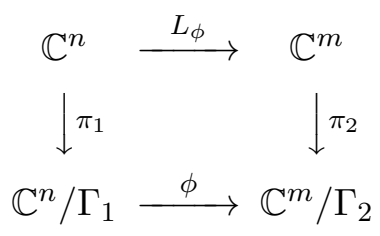


where $\pi_{1}, \pi_{2}$ are the natural projections. Conversely, given such a $\mathbb{C}$-linear map, it gives by passage to quotient a unique morphism of connected abelian complex Lie groups.

Proof. There are two points of view. The first one is to see $\pi_{i}$ as the corresponding exponential map in which case $L_{\phi}$ is given by the basic theory of Lie groups. The second one consists in viewing $\pi_{i}$ as the corresponding universal covering map and then use the lifting theorem plus the fact that a holomorphic group homomorphism from $\mathbb{C}^{n}$ to $\mathbb{C}^{m}$ is necessarily $\mathbb{C}$-linear.

Because of Lemma 2, one can associate to $\mathbb{C}^{n} / \Gamma$ two representations of End $\left(\mathbb{C}^{n} / \Gamma\right)$. The first one is the analytic representation

$$
\rho_{a}: \operatorname{End}\left(\mathbb{C}^{n} / \Gamma\right) \rightarrow \operatorname{End}_{\mathbb{C}}\left(\mathbb{C}^{n}\right),
$$

defined by $\phi \mapsto L_{\phi}$. The second one is the rational representation: Note that $L_{\phi}(\Gamma) \subseteq \Gamma$ for all $\phi \in \operatorname{End}\left(\mathbb{C}^{n} / \Gamma\right)$ and therefore, we can consider $L_{\phi}$ as an endomorphism of the free abelian group $\Gamma$. In this way, we get the rational representation

$$
\rho_{r}: \operatorname{End}\left(\mathbb{C}^{n} / \Gamma\right) \rightarrow \operatorname{End}_{\mathbb{Z}}(\Gamma),
$$

defined by $\phi \mapsto L_{\phi}$.

Now, if we start with a connected abelian complex Lie group $G$ and we choose a lattice representation

$$
\xi: G \rightarrow \mathbb{C}^{n} / \Gamma
$$

then the compositions $\rho_{a} \circ \tilde{\xi}$ and $\rho_{r} \circ \tilde{\xi}$ give representations of $\operatorname{End}(G)$. What happens if we choose another lattice representation? Well, it is not hard to check that the corresponding representations are isomorphic.

Lemma 3. It is clear from the uniqueness in Lemma 2 that the analytic representation of $\mathbb{C}^{n} / \Gamma$ is faithful. If we suppose moreover that $\operatorname{rank}_{\mathbb{C}}(\Gamma)=$ $n$, then the rational representation is also faithful.

Proof. If $L_{\phi_{1}}=L_{\phi_{2}}$ on $\Gamma$, we get that $L_{\phi_{1}}=L_{\phi_{2}}$ on $\mathbb{C}^{n}$ since $\operatorname{Span}_{\mathbb{C}}(\Gamma)=$ $\mathbb{C}^{n}$ and therefore $\phi_{1}=\phi_{2}$.

Remark. Note that this last lemma applies to Cousin groups.

Corollary 2. Let $G$ be a connected abelian complex Lie group of complex dimension $n$, and suppose that $\operatorname{rank}_{\mathbb{C}}(G)=n$, and $\operatorname{rank}(G)=n+m$, then $\operatorname{End}(G)$ is a free abelian group of rank smaller or equal to $(n+m)^{2}$.

For the sake of conciseness, we shall denote $\mathbb{Q} \otimes_{\mathbb{Z}} \operatorname{End}(G)$ by $\operatorname{End}_{0}(G)$. After tensoring with $\mathbb{Q}$ we get representations of $\operatorname{End}_{0}\left(\mathbb{C}^{n} / \Gamma\right)$ which we denote by the same symbols:

$$
\rho_{a}: \operatorname{End}_{0}\left(\mathbb{C}^{n} / \Gamma\right) \rightarrow \operatorname{End}_{\mathbb{C}}\left(\mathbb{C}^{n}\right),
$$


and

$$
\rho_{r}: \operatorname{End}_{0}\left(\mathbb{C}^{n} / \Gamma\right) \rightarrow \operatorname{End}_{\mathbb{Q}}\left(\operatorname{Span}_{\mathbb{Q}}(\Gamma)\right) .
$$

Moreover, the associated matrix representation, after we choose the canonical basis of $\mathbb{C}^{n}$, will be denoted by $\phi \mapsto M_{\phi}$.

Corollary 3. Let $G$ be a connected abelian complex Lie group of complex dimension $n$, and suppose that $\operatorname{rank}_{\mathbb{C}}(G)=n$, and that $\operatorname{rank}(G)=n+m$, then $\operatorname{End}_{0}(G)$ is a finite dimensional $\mathbb{Q}$-algebra of dimension smaller or equal to $(n+m)^{2}$.

It would be interesting to have some general structure theorems about these finite dimensional $\mathbb{Q}$-algebras. Particular cases have been worked out but, as far as we know, the answer in general is not known.

Note also that given any connected abelian complex Lie group, there exist some trivial endomorphisms which are just multiplication by $n$ (denoted by $[n])$. It is clear that the map

$$
[n]: G \rightarrow G,
$$

given by $g \mapsto n \cdot g$ is a group homomorphism. Moreover, it is also holomorphic. Indeed, if $n=2$, it is holomorphic because it is the composition of two holomorphic maps, namely the diagonal map and the group law itself. An induction then shows that the maps $[n]$ are holomorphic for all $n \geq 1$. Since the inverse map is also holomorphic, we conclude that $[n]$ is an endomorphism in the category of complex abelian Lie groups for all $n \in \mathbb{Z}$. Hence, $\operatorname{End}(G)$ always contains a subring isomorphic to $\mathbb{Z}$. For most of them, this is the end of the story. On the other hand, those having a ring of endomorphisms strictly bigger than $\mathbb{Z}$ seem to have some connections with arithmetic.

2.2. Matrix notation. Let $\mathbb{C}^{n} / \Gamma$ be a connected abelian complex Lie group of rank $t$. When it is time to prove something particular about a given connected abelian complex Lie group, it is often useful to express everything in terms of matrices. Suppose that $\gamma_{1}, \ldots, \gamma_{t}$ is a basis for the lattice $\Gamma$. Suppose also that with respect to the canonical basis $\left(e_{1}, \ldots, e_{n}\right)$ for $\mathbb{C}^{n}$ the coordinates of $\gamma_{j}$ are given by

$$
\gamma_{j}=a_{1 j} e_{1}+\ldots+a_{n j} e_{n}
$$

then the matrix

$$
\Omega=\left(a_{i j}\right) \in \mathrm{M}_{n \times t}(\mathbb{C})
$$

will be called a period matrix of $\Gamma$.

If we are given a $\phi \in \operatorname{End}\left(\mathbb{C}^{n} / \Gamma\right)$ and if $M=M_{\phi}$ is the corresponding matrix representing $L_{\phi}$ in the canonical basis of $\mathbb{C}^{n}$, then there exists a matrix $A \in \mathrm{M}_{t}(\mathbb{Z})$ such that

$$
M \Omega=\Omega A
$$


These are the so-called Hurwitz relations. The matrices $A \in M_{t}(\mathbb{Z})$ are called multipliers of $\Omega$ and the matrices $M_{\phi} \in M_{n}(\mathbb{C})$ are called multiplications of $\Omega$.

Lemma 4. Let $\mathbb{C}^{n} / \Gamma$ be a connected abelian complex Lie group of rank $n+m$ with $m \geq 1$ and such that $\operatorname{rank}_{\mathbb{C}}(\Gamma)=n$. One can find a basis for which the period matrix of $\Gamma$ has the form

$$
\left(\begin{array}{ccc}
I_{n-m} & \tau_{1} & \tau_{2} \\
0 & I_{m} & \tau
\end{array}\right)
$$

where $\tau_{1}, \tau_{2} \in M_{n-m, m}(\mathbb{R}), \tau \in M_{m}(\mathbb{C})$ and $\left(I_{m} \tau\right)$ is the period matrix of a complex torus of dimension $m$ and $\operatorname{Im}(\tau)$ has rank $m$.

Proof. Because of Lemma 1, we can assume that the lattice looks like $\mathbb{Z}^{n} \oplus \Lambda$, where $\Lambda$ is another lattice such that $\operatorname{Im}(\Lambda)$ has rank $m$. We can thus find a basis for which the period matrix looks like

$$
\left(\begin{array}{ccc}
I_{n-m} & 0 & \alpha_{1} \\
0 & I_{m} & \alpha_{2}
\end{array}\right)
$$

where $\alpha_{1} \in M_{n-m, m}(\mathbb{C})$ and $\alpha_{2} \in M_{m}(\mathbb{C})$. Since $\operatorname{Im}(\Lambda)$ has rank $m$, we can also suppose that $\operatorname{Im}\left(\alpha_{2}\right)$ is invertible. Consider then the matrix

$$
\left(\begin{array}{cc}
I_{n-m} & -\operatorname{Im}\left(\alpha_{1}\right) \operatorname{Im}\left(\alpha_{2}\right)^{-1} \\
0 & I_{m}
\end{array}\right)
$$

which is clearly in $\mathrm{GL}_{n}(\mathbb{R})$. We have

$$
\left(\begin{array}{cc}
I_{n-m} & -\operatorname{Im}\left(\alpha_{1}\right) \operatorname{Im}\left(\alpha_{2}\right)^{-1} \\
0 & I_{m}
\end{array}\right) \cdot\left(\begin{array}{ccc}
I_{n-m} & 0 & \alpha_{1} \\
0 & I_{m} & \alpha_{2}
\end{array}\right)=\left(\begin{array}{ccc}
I_{n-m} & \tau_{1} & \tau_{2} \\
0 & I_{m} & \tau
\end{array}\right)
$$

where

$$
\tau_{1}=-\operatorname{Im}\left(\alpha_{1}\right) \operatorname{Im}\left(\alpha_{2}\right)^{-1} \in \mathrm{M}_{n-m, m}(\mathbb{R}),
$$

and

$$
\tau_{2}=\alpha_{1}-\operatorname{Im}\left(\alpha_{1}\right) \operatorname{Im}\left(\alpha_{2}\right)^{-1} \alpha_{2} \in \mathrm{M}_{n-m, m}(\mathbb{R}) .
$$

Moreover, $\tau=\alpha_{2}$ and since $\operatorname{Im}\left(\alpha_{2}\right)$ has rank $m$ we conclude that $\left(I_{m} \tau\right)$ is indeed the period matrix of a complex torus. This is what we wanted to prove.

Remark. For a Cousin group, these particular basis elements for which the period matrix looks like the one above are usually called toroidal coordinates in the literature. They are also called apt coordinates in [1].

If the period matrix of a connected abelian complex Lie group has the shape discribed in the last lemma, then the irrationality condition (Theorem 2) becomes quite simple (the proof is left to the reader): 
Lemma 5. Suppose that $\mathbb{C}^{n} / \Gamma$ is a connected abelian complex Lie group of rank $n+m$, with $m \geq 1$ and suppose also that the period matrix associated with $\Gamma$ has the form

$$
\left(\begin{array}{ccc}
I_{n-m} & \tau_{1} & \tau_{2} \\
0 & I_{m} & \tau
\end{array}\right)
$$

where $\tau_{1}, \tau_{2} \in M_{n-m, m}(\mathbb{R}), \tau \in M_{m}(\mathbb{C})$ and $\left(I_{m} \tau\right)$ is the period matrix of a complex torus of dimension $m$. Then $\mathbb{C}^{n} / \Gamma$ is a Cousin group if and only if given any $s \in \mathbb{Z}^{n-m} \backslash\{0\}$, one has

$$
s \cdot\left(\tau_{1} \tau_{2}\right) \notin \mathbb{Z}^{2 m} .
$$

\section{Non-totally real cubic number fields}

In the theory of explicit class field theory for $\mathbb{Q}$ and also for $C M$-fields, there are connected abelian complex Lie groups which are somehow at the origin of any arithmetical investigation. These analytico-geometrical objects can be realized with the help of the Minkowski map, and similar objects can be constructed for any number field, not only for $\mathbb{Q}$ and for $C M$-fields. We start by explaining this.

Definition 6. Let $K$ be a number field, and let $r=r_{1}+r_{2}$, where as usual $r_{1}$ (resp. $2 r_{2}$ ) is the number of real (resp. complex) embeddings of $K$. A complete set $\Phi=\left\{\sigma_{1}, \ldots, \sigma_{r}\right\}$ of representatives modulo complex conjugation for the embeddings of $K$ into $\mathbb{C}$ where the first $r_{1}$ embeddings are real will be called a type.

Remark. If $K$ is a $C M$-field, a type is usually called a $C M$-type.

To any type $\Phi$ for $K$, one can associate the Minkowski map

$$
\mu_{\Phi}: K \rightarrow \mathbb{C}^{r}
$$

defined by $\lambda \mapsto\left(\sigma_{1}(\lambda), \ldots, \sigma_{r}(\lambda)\right)$.

Theorem 4. Let $K$ be a number field of degree $n$ over $\mathbb{Q}$ and let $\Phi$ be a type of $K$. Given any free $\mathbb{Z}$-module $\mathfrak{m}$ of rank $n$ in $K$, the free $\mathbb{Z}$-module $\mu_{\Phi}(\mathfrak{m})$ is in fact a lattice in $\mathbb{C}^{r}$. Moreover

- $\operatorname{rank}\left(\mu_{\Phi}(\mathfrak{m})\right)=r_{1}+2 r_{2}=[K: \mathbb{Q}]$,

- $\operatorname{rank}_{\mathbb{C}}\left(\mu_{\Phi}(\mathfrak{m})\right)=r_{1}+r_{2}=r$.

Proof. Indeed, suppose that a $\mathbb{Z}$-basis for $\mathfrak{m}$ is given by $\left(\beta_{1}, \ldots, \beta_{n}\right)$, then this basis is in fact a $\mathbb{Q}$-basis for the $\mathbb{Q}$-vector space $K$ (this can be checked easily since $\mathbb{Q}$-linearly dependence implies $\mathbb{Z}$-linearly dependence). Therefore, we have

$$
\operatorname{disc}\left(\beta_{1}, \ldots, \beta_{n}\right) \neq 0,
$$

by a classical result in algebraic number theory. In order to show that $\mu_{\Phi}(\mathfrak{m})$ is a lattice, we just have to show that the vectors $\mu_{\Phi}\left(\beta_{1}\right), \ldots, \mu_{\Phi}\left(\beta_{n}\right)$ are 
$\mathbb{R}$-linearly independent. Suppose they are not, then there exist numbers $\lambda_{1}, \ldots, \lambda_{n} \in \mathbb{R}$ not all zero such that

$$
\lambda_{1} \mu_{\Phi}\left(\beta_{1}\right)+\ldots+\lambda_{n} \mu_{\Phi}\left(\beta_{n}\right)=0 .
$$

Then we would get in column notation

$$
\lambda_{1}\left(\begin{array}{c}
\sigma_{1}\left(\beta_{1}\right) \\
\vdots \\
\sigma_{r_{1}}\left(\beta_{1}\right) \\
\sigma_{r_{1}+1}\left(\beta_{1}\right) \\
\vdots \\
\sigma_{r_{1}+r_{2}}\left(\beta_{1}\right) \\
\sigma_{r_{1}+r_{2}+1}\left(\beta_{1}\right) \\
\vdots \\
\sigma_{r_{1}+2 r_{2}}\left(\beta_{1}\right)
\end{array}\right)+\ldots+\lambda_{n}\left(\begin{array}{c}
\sigma_{1}\left(\beta_{n}\right) \\
\vdots \\
\sigma_{r_{1}}\left(\beta_{n}\right) \\
\sigma_{r_{1}+1}\left(\beta_{n}\right) \\
\vdots \\
\sigma_{r_{1}+r_{2}}\left(\beta_{n}\right) \\
\sigma_{r_{1}+r_{2}+1}\left(\beta_{n}\right) \\
\vdots \\
\sigma_{r_{1}+2 r_{2}}\left(\beta_{n}\right)
\end{array}\right)=0
$$

where $\sigma_{j}=\bar{\sigma}_{j-r_{2}}$ for $j=r_{1}+r_{2}+1, \ldots, r_{1}+2 r_{2}$. This would contradict the fact that

$$
\operatorname{disc}\left(\beta_{1}, \ldots, \beta_{n}\right) \neq 0 \text {. }
$$

We also conclude that

$$
\operatorname{rank}\left(\mu_{\Phi}(\mathfrak{m})\right)=n=[K: \mathbb{Q}] .
$$

Moreover, since the matrix $\left(\sigma_{i}\left(\beta_{j}\right)\right)$ has full rank we conclude as well that

$$
\operatorname{rank}_{\mathbb{C}}\left(\mu_{\Phi}(\mathfrak{m})\right)=r=r_{1}+r_{2}
$$

Thus, from any free $\mathbb{Z}$-module $\mathfrak{m}$ of rank $n$ in $K$, we get a connected abelian complex Lie group $\mathbb{C}^{r} / \mu_{\Phi}(\mathfrak{m})$. But these are really special connected abelian complex Lie groups since they are built out of some arithmetical data.

From now on, we shall focus on the case where $K$ is a cubic number field which is not totally real; such a field cannot be Galois. We will also only work with fractional ideals, even though some results might be true more generally for any module of $K$. We do this only for the sake of simplicity.

3.1. The Remmert-Morimoto decomposition. Let $K$ be a number field, $\Phi$ a type of $K$ and let also $\mathfrak{a}$ be a fractional ideal in $K$. The first question one might ask is: What is the Remmert-Morimoto decomposition of $\mathbb{C}^{r} / \mu_{\Phi}(\mathfrak{a})$ ? If $K$ is totally real, then Corollary 1 and Theorem 4 show that $\mathbb{C}^{r} / \mu_{\Phi}(\mathfrak{a}) \simeq\left(\mathbb{C}^{\times}\right)^{r_{1}}$. If $K$ is a non-totally real cubic number field, we have the following theorem. 
Theorem 5. Let $K$ be a non-totally real cubic number field and $\mathfrak{a}$ a fractional ideal of $K$. The connected abelian complex Lie group $\mathbb{C}^{2} / \mu_{\Phi}(\mathfrak{a})$ is a Cousin group.

Proof. We use Theorem 2. Let $L \in \operatorname{Hom}_{\mathbb{C}}\left(\mathbb{C}^{2}, \mathbb{C}\right)$ be such that $L\left(\mu_{\Phi}(\mathfrak{a})\right) \subseteq$ $\mathbb{Z}$. Write also $\mathfrak{a}=\mathbb{Z} \alpha_{1}+\mathbb{Z} \alpha_{2}+\mathbb{Z} \alpha_{3}$ for some $\alpha_{i} \in K$ and where the sum is direct. Note again that the $\alpha_{i}$ are $\mathbb{Q}$-linearly independent. Let us denote the canonical basis of $\mathbb{C}^{2}$ by $\left(e_{1}, e_{2}\right)$. Then the matrix corresponding to $L$ will be denoted by $\left(z_{1} z_{2}\right)$ for some $z_{i} \in \mathbb{C}$ which cannot be both zero. The hypothesis $L\left(\mu_{\Phi}(\mathfrak{a})\right) \subseteq \mathbb{Z}$ implies that there exists $n_{1}, n_{2}, n_{3} \in \mathbb{Z}$ such that in matrix notation, we have

$$
\left(\begin{array}{ll}
\sigma_{1}\left(\alpha_{1}\right) & \sigma_{2}\left(\alpha_{1}\right) \\
\sigma_{1}\left(\alpha_{2}\right) & \sigma_{2}\left(\alpha_{2}\right) \\
\sigma_{1}\left(\alpha_{3}\right) & \sigma_{2}\left(\alpha_{3}\right)
\end{array}\right)\left(\begin{array}{l}
z_{1} \\
z_{2}
\end{array}\right)=\left(\begin{array}{l}
n_{1} \\
n_{2} \\
n_{3}
\end{array}\right) .
$$

Taking complex conjugation of this last equation, we obtain

$$
\left(\begin{array}{ll}
\sigma_{1}\left(\alpha_{1}\right) & \overline{\sigma_{2}}\left(\alpha_{1}\right) \\
\sigma_{1}\left(\alpha_{2}\right) & \overline{\sigma_{2}}\left(\alpha_{2}\right) \\
\sigma_{1}\left(\alpha_{3}\right) & \overline{\sigma_{2}}\left(\alpha_{3}\right)
\end{array}\right)\left(\begin{array}{l}
\bar{z}_{1} \\
\bar{z}_{2}
\end{array}\right)=\left(\begin{array}{l}
n_{1} \\
n_{2} \\
n_{3}
\end{array}\right) .
$$

Subtracting Equation 3.2 from Equation 3.1 we get

$$
\left(\begin{array}{lll}
\sigma_{1}\left(\alpha_{1}\right) & \sigma_{2}\left(\alpha_{1}\right) & \overline{\sigma_{2}}\left(\alpha_{1}\right) \\
\sigma_{1}\left(\alpha_{2}\right) & \sigma_{2}\left(\alpha_{2}\right) & \overline{\sigma_{2}}\left(\alpha_{2}\right) \\
\sigma_{1}\left(\alpha_{3}\right) & \sigma_{2}\left(\alpha_{3}\right) & \overline{\sigma_{2}}\left(\alpha_{3}\right)
\end{array}\right)\left(\begin{array}{c}
z_{1}-\bar{z}_{1} \\
z_{2} \\
-\bar{z}_{2}
\end{array}\right)=\left(\begin{array}{l}
0 \\
0 \\
0
\end{array}\right),
$$

but the determinant of the $3 \times 3$ matrix is non-zero and therefore, we conclude that $z_{1} \in \mathbb{R}$ and $z_{2}=0$. Hence we are just left with the equation

$$
z_{1}\left(\begin{array}{l}
\sigma_{1}\left(\alpha_{1}\right) \\
\sigma_{1}\left(\alpha_{2}\right) \\
\sigma_{1}\left(\alpha_{3}\right)
\end{array}\right)=\left(\begin{array}{l}
n_{1} \\
n_{2} \\
n_{3}
\end{array}\right) .
$$

If $z_{1} \neq 0$ we would have

$$
n_{2} \sigma_{1}\left(\alpha_{1}\right)=n_{1} \sigma_{1}\left(\alpha_{2}\right),
$$

that is

$$
\sigma_{1}\left(n_{2} \alpha_{1}-n_{1} \alpha_{2}\right)=0 .
$$

Since the $\alpha_{i}$ are $\mathbb{Q}$-linearly independent, we conclude that $n_{1}=n_{2}=0$ and since in particular $\sigma_{1}\left(\alpha_{1}\right) \neq 0$, one gets $z_{1}=0$. Hence, $z_{1}=0$ necessarily and $L$ is the trivial map. By Theorem 2, we see that $\mathbb{C}^{2} / \mu_{\Phi}(\mathfrak{a})$ is Cousin.

Remark. As noted by the referee, the same proof works for any number field satisfying $r_{1}=1$ and $r_{2} \geq 1$. 
3.2. The ring of endomorphisms. In this section, we study the structure of $\operatorname{End}(G)$ and $\operatorname{End}_{0}(G)$ for Cousin groups and especially those of complex dimension 2 and rank 3 . Recall, that we always have $\mathbb{Z} \subseteq \operatorname{End}(G)$, since we have the multiplication-by- $n$ endomorphisms denoted by $[n]$.

Definition 7. Let $G$ be a Cousin group. We shall say that $G$ admits extra multiplication if $\mathbb{Z}$ is strictly included in $\operatorname{End}(G)$ but not equal to it.

Lemma 6. Let $\mathbb{C}^{n} / \Gamma$ be a Cousin group of rank $n+1$. Suppose that $\Gamma=$ $\Gamma_{1} \oplus \Gamma_{2}$ where $\operatorname{rank}_{\mathbb{Z}}\left(\Gamma_{1}\right)=n$ and $\operatorname{rank}_{\mathbb{Z}}\left(\Gamma_{2}\right)=1$, then $\operatorname{rank}_{\mathbb{C}}\left(\Gamma_{1}\right)=n$.

Proof. Let $W_{i}=\operatorname{Span}_{\mathbb{C}}\left(\Gamma_{i}\right)$ for $i=1,2$ and suppose that $\operatorname{rank}_{\mathbb{C}}\left(\Gamma_{1}\right)<n$. Since $\mathbb{C}^{n} / \Gamma$ is Cousin, we have that $W_{1}+W_{2}=\mathbb{C}^{n}$. Moreover, $\operatorname{dim}_{\mathbb{C}}\left(W_{2}\right)=$ 1. Using the well-known formula

$$
\operatorname{dim}\left(W_{1}+W_{2}\right)=\operatorname{dim}\left(W_{1}\right)+\operatorname{dim}\left(W_{2}\right)-\operatorname{dim}\left(W_{1} \cap W_{2}\right),
$$

we get

$$
\operatorname{dim}\left(W_{1} \cap W_{2}\right)=1+\operatorname{dim}_{\mathbb{C}}\left(W_{1}\right)-n<1+n-n=1 .
$$

Thus, $W_{1} \cap W_{2}=\{0\}$ and $\mathbb{C}^{n}=W_{1} \oplus W_{2}$. But then we get

$$
\mathbb{C}^{n} / \Gamma \simeq W_{1} / \Gamma_{1} \times W_{2} / \Gamma_{2}
$$

Since the second factor is isomorphic to $\mathbb{C}^{\times}$, we get a contradiction because $\mathbb{C}^{n} / \Gamma$ is Cousin.

Lemma 7. Let $\mathbb{C}^{n} / \Gamma$ be a Cousin group of rank $n+1$ and $\phi \in \operatorname{End}\left(\mathbb{C}^{n} / \Gamma\right)$. If $\phi \neq 0$, then $L_{\phi} \in \mathrm{GL}_{\mathbb{C}}\left(\mathbb{C}^{n}\right)$.

Proof. If $L_{\phi}$ is not surjective then $\operatorname{rank}_{\mathbb{Z}}\left(L_{\phi}(\Gamma)\right) \leq n$. This rank cannot be zero either, otherwise $L_{\phi}=0$ which is excluded. One can then find a basis $\gamma_{1}, \ldots, \gamma_{n+1}$ of $\Gamma$ and positive integers $t_{1}, \ldots, t_{s},(s \leq n)$, such that $t_{1} \gamma_{1}, \ldots, t_{s} \gamma_{s}$ is a basis for $L_{\phi}(\Gamma)$. By Lemma $6, \gamma_{1}, \ldots, \gamma_{s}$ are $\mathbb{C}$ linearly independent. Let $W=\operatorname{Span}_{\mathbb{C}}\left(L_{\phi}(\Gamma)\right)$ and take any $\mathbb{C}$-linear map $T \in \operatorname{Hom}_{\mathbb{C}}(W, \mathbb{C})$ which satisfies $T\left(\gamma_{i}\right)=1$ for $i=1, \ldots, s$. Then $T \circ$ $L_{\phi}$ is a non-zero linear form on $\mathbb{C}^{n}$ taking integer values on $\Gamma$. This is a contradiction.

Corollary 4. Let $G$ be a Cousin group of complex dimension $n$ and of rank $n+1$ and let also $\phi \in \operatorname{End}(G)$. If $\phi \neq 0$ then $\phi$ is surjective.

Proof. Let $\xi: G \rightarrow \mathbb{C}^{n} / \Gamma$ be a lattice representation of $G$. We just have to show the corresponding fact for $\mathbb{C}^{n} / \Gamma$, so if $\phi \in \operatorname{End}\left(\mathbb{C}^{n} / \Gamma\right)$ is such, then Lemma 7 implies that $L_{\phi} \in \mathrm{GL}_{\mathbb{C}}\left(\mathbb{C}^{n}\right)$ if $\phi \neq 0$. This implies that $\phi$ itself is surjective.

Lemma 8. Let $G$ be a Cousin group of complex dimension $n$ and of rank $n+1$. Let also $\phi \in \operatorname{End}(G)$ and suppose that $\phi \neq 0$, then $\operatorname{ker}(\phi)$ is finite. 
Proof. Again, we choose a lattice representation $\xi: G \rightarrow \mathbb{C}^{n} / \Gamma$ and we just have to prove the corresponding fact for endomorphisms of $\mathbb{C}^{n} / \Gamma$. We saw that if $\phi \neq 0$ then $L_{\phi}$ is in fact an isomorphism of $\mathbb{C}$-vector space. Moreover, we have

$$
\operatorname{ker}(\phi) \simeq L_{\phi}^{-1}(\Gamma) / \Gamma .
$$

Since $L_{\phi}$ is an isomorphism, we get that $L_{\phi}^{-1}(\Gamma)$ is also a free abelian group of rank $n+1$, and we conclude that the kernel is finite.

We saw that any nontrivial endomorphism of $G$ is surjective and has finite kernel. As in the classical case of complex tori, we shall call such a map an isogeny.

Theorem 6. Let $G$ be a Cousin group of complex dimension $n$ and rank $n+1$ and let $\phi \in \operatorname{End}(G)$ and suppose that $\phi \neq 0$. Let $e$ be the exponent of the finite group $\operatorname{ker}(\phi)$, then there exists another $\psi \in \operatorname{End}(G)$ such that

- $\phi \circ \psi=[e]$,

- $\psi \circ \phi=[e]$.

Proof. Since $\operatorname{ker}(\phi) \subseteq \operatorname{ker}([e])$ we get a morphism $\psi \in \operatorname{End}(G)$ satisfying

$$
\psi \circ \phi=[e] \text {. }
$$

Now we claim that $\operatorname{ker}(\psi) \subseteq \operatorname{ker}([e])$. This is clear because if $x \in \operatorname{ker}(\psi)$, then there exists $y \in \operatorname{ker}([e])$ such that $\phi(y)=x$, since

$$
\operatorname{ker}([e])=\phi^{-1}(\operatorname{ker}(\psi)) \text {. }
$$

We then get

$$
[e](x)=[e](\phi(y))=\phi([e](y))=0 .
$$

Hence, there exists a $\phi^{\prime} \in \operatorname{End}(G)$ satisfying

$$
\phi^{\prime} \circ \psi=[e] .
$$

We would like to show that $\phi=\phi^{\prime}$, but this is indeed the case since

$$
\phi^{\prime} \circ[e]=\phi^{\prime} \circ \psi \circ \phi=[e] \circ \phi=\phi \circ[e],
$$

and we can conclude the desired equality because $[e]$ is surjective.

Theorem 7. Given any Cousin group $G$ of complex dimension $n$ and of rank $n+1$, we have that $\operatorname{End}_{0}(G)$ is a division algebra, where we remind the reader that a division algebra is an algebra such that every non-zero element has a multiplicative inverse.

Proof. This is immediate from Theorem 6.

Lemma 9. Let $A$ be a non-trivial finite dimensional $\mathbb{Q}$-algebra and suppose it is a division algebra. Suppose also that we have a linear representation

$$
\rho: A \rightarrow \operatorname{End}_{\mathbb{Q}}(V),
$$


into a finite dimensional $\mathbb{Q}$-vector space. Then, we necessarily have

$$
\operatorname{dim}_{\mathbb{Q}}(A) \mid \operatorname{dim}_{\mathbb{Q}}(V) .
$$

Proof. Note first that $\rho$ has to be injective since $A$ is a division algebra. Hence we can identified $A$ with $\rho(A)$. We have that $V$ becomes an $A$-vector space, the action of $A$ being given by

$$
a \cdot v:=\rho(a)(v) .
$$

We then have the following following formula relating the different dimensions:

$$
\operatorname{dim}_{\mathbb{Q}}(V)=\operatorname{dim}_{\mathbb{Q}}(A) \cdot \operatorname{dim}_{A}(V) .
$$

We conclude immediately that

$$
\operatorname{dim}_{\mathbb{Q}}(A) \mid \operatorname{dim}_{\mathbb{Q}}(V) .
$$

We now come back to non-totally real cubic number fields and Cousin groups of complex dimension 2 and rank 3 .

Corollary 5. Given any Cousin group $G$ of complex dimension 2 and of rank 3 , we have

$$
\operatorname{dim}_{\mathbb{Q}}\left(\operatorname{End}_{0}(G)\right)=1 \text { or } 3 .
$$

Proof. Take a lattice representation $\xi: G \rightarrow \mathbb{C}^{2} / \Gamma$. Apply Lemma 9 with $A=\operatorname{End}_{0}\left(\mathbb{C}^{2} / \Gamma\right)$ and $\rho=\rho_{r}$ the rational representation.

Corollary 6. Let $G$ be a Cousin group of complex dimension 2 and of rank 3. If $G$ has extra multiplication, then $\operatorname{End}_{0}(G)$ is a cubic number field, and $\operatorname{End}(G)$ is an order in this cubic number field.

Proof. If $\operatorname{End}_{0}(G) \neq \mathbb{Q}$ we conclude that it is of dimension 3 over $\mathbb{Q}$ by Corollary 5. Now it is known that the dimension of a simple algebra over its center is a square. Hence this dimension has to be one. This shows that $A$ is commutative and hence a cubic number field.

Note then that since $\operatorname{End}(G)$ is without torsion, we have an injective morphism

$$
\operatorname{End}(G) \hookrightarrow \operatorname{End}_{0}(G) .
$$

We know that $\operatorname{End}(G)$ is a free abelian group, but since $\operatorname{End}_{0}(G)$ is of dimension 3 over $\mathbb{Q}$, we see that the rank of $\operatorname{End}(G)$ has to be 3 . Now, because the map above is injective, we get that $\operatorname{End}(G)$ is a subring of a cubic number field which is at the same time a free abelian group of rank 3 . This is precisely the definition of an order in a number field.

We shall now show that in the case of extra multiplication, $\operatorname{End}_{0}(G)$ cannot be totally real: It has to satisfy $r_{1}=1=r_{2}$. How could one show such a thing? We have to find how to produce embeddings of this number field into $\mathbb{C}$. This has to be related with the analytic representation. 
Theorem 8. If the Cousin group $G$ of complex dimension 2 and of rank 3 has extra multiplication, then $\operatorname{End}_{0}(G)$ is necessarily a cubic number field which is not totally real.

Proof. Let $\xi: G \rightarrow \mathbb{C}^{2} / \Gamma$ be a lattice representation. Because of Lemma 4 , we can also suppose that the period matrix of $\Gamma$ has the form

$$
\Omega=\left(\begin{array}{ccc}
1 & \tau_{1} & \tau_{2} \\
0 & 1 & \tau
\end{array}\right)
$$

where $\tau_{1}, \tau_{2} \in \mathbb{R}$ and $\operatorname{Im}(\tau) \neq 0$. Let us denote $\operatorname{End}_{0}\left(\mathbb{C}^{2} / \Gamma\right)$ by $K$ and End $\left(\mathbb{C}^{2} / \Gamma\right)$ by $O$. We know that $O$ is an order in $K$. Let us choose also the canonical basis $\left(e_{1}, e_{2}\right)$ of $\mathbb{C}^{2}$. Then the analytic representation becomes a matrix representation $O \rightarrow \mathrm{M}_{2}(\mathbb{C})$ which we shall denote by

$$
\lambda \rightarrow M(\lambda)=\left(m_{i j}(\lambda)\right) .
$$

If we write the multiplications in terms of matrices, we get the Hurwitz relations:

$$
M(\lambda) \cdot \Omega=\Omega \cdot A(\lambda), \text { for all } \lambda \in O,
$$

where $A(\lambda)=\left(a_{i j}(\lambda)\right)$ is some matrix in $\mathrm{M}_{3}(\mathbb{Z})$. If we expand the Hurwitz relations, we get the following matrix equality:

$$
\begin{aligned}
& \left(\begin{array}{lll}
m_{11} & m_{11} \tau_{1}+m_{12} & m_{11} \tau_{2}+m_{12} \tau \\
m_{21} & m_{21} \tau_{1}+m_{22} & m_{21} \tau_{2}+m_{22} \tau
\end{array}\right)= \\
& \left(\begin{array}{ccc}
a_{11}+a_{21} \tau_{1}+a_{31} \tau_{2} & a_{12}+a_{22} \tau_{1}+a_{32} \tau_{2} & a_{13}+a_{23} \tau_{1}+a_{33} \tau_{2} \\
a_{21}+a_{31} \tau & a_{22}+a_{32} \tau & a_{23}+a_{33} \tau
\end{array}\right),
\end{aligned}
$$

where we drop the $\lambda$ 's for the ease of reading. Now looking at the $(1,1)$ entry of these two matrices we get that $m_{11}(\lambda) \in \mathbb{R}$ for all $\lambda \in O$. Then, looking at the $(1,2)$-entry of these two matrices, we get that $m_{12}(\lambda) \in \mathbb{R}$ for all $\lambda \in O$. But then looking at the $(1,3)$-entry, we get the following equation

$$
m_{12}(\lambda)(\tau-\bar{\tau})=0
$$

and therefore, we conclude that $m_{12}(\lambda)=0$ for all $\lambda \in O$ since $\operatorname{Im}(\tau) \neq 0$.

Therefore the matrix representation associated to the analytic representation has the form

$$
\lambda \mapsto M(\lambda)=\left(\begin{array}{cc}
m_{11}(\lambda) & 0 \\
m_{21}(\lambda) & m_{22}(\lambda)
\end{array}\right),
$$

where $m_{11}(\lambda) \in \mathbb{R}$ for all $\lambda \in O$. Since the matrix representation is in particular a ring homomorphism, we get

$$
M\left(\lambda_{1} \lambda_{2}\right)=M\left(\lambda_{1}\right) M\left(\lambda_{2}\right),
$$

for all $\lambda_{1}, \lambda_{2} \in O$. This implies that the maps

$$
\lambda \mapsto m_{11}(\lambda),
$$


and

$$
\lambda \mapsto m_{22}(\lambda),
$$

are in fact ring homomorphisms of $O$ into $\mathbb{C}$ and they induce field embeddings of $K$ into $\mathbb{C}$ which we shall denote by the same symbol. Moreover $m_{11}$ is a real embedding. We shall now show that $m_{22}$ has to be a complex embedding and this will end the proof that $K$ is not totally real. Suppose that $m_{22}(\lambda) \in \mathbb{R}$ for all $\lambda \in O$, then looking at the second line of the matrix equality above, we get the following three equations:

$$
\begin{gathered}
m_{21}=a_{21}+a_{31} \tau \\
m_{21} \tau_{1}+m_{22}=a_{22}+a_{32} \tau \\
m_{21} \tau_{2}+m_{22} \tau=a_{23}+a_{33} \tau
\end{gathered}
$$

Multiplying Equation 3.3 by $\tau_{1}$ gives

$$
m_{21} \tau_{1}=a_{21} \tau_{1}+a_{31} \tau \tau_{1} .
$$

Subtracting Equation 3.4 from this last equation, we get

$$
a_{22}-a_{21} \tau_{1}-m_{22}=\tau\left(a_{31} \tau_{1}-a_{32}\right) \text {. }
$$

Multiplying Equation 3.3 by $\tau_{2}$, we get

$$
m_{21} \tau_{2}=a_{21} \tau_{2}+a_{31} \tau \tau_{2} .
$$

Subtracting Equation 3.5 from this last equation, we get

$$
a_{23}-a_{21} \tau_{2}=\tau\left(a_{31} \tau_{2}-a_{33}+m_{22}\right) \text {. }
$$

Multiplying Equation 3.4 by $\tau_{2}$, we get

$$
m_{21} \tau_{1} \tau_{2}+m_{22} \tau_{2}=a_{22} \tau_{2}+a_{32} \tau \tau_{2} .
$$

Multiplying Equation 3.5 by $\tau_{1}$, we get

$$
m_{21} \tau_{2} \tau_{1}+m_{22} \tau \tau_{1}=a_{23} \tau_{1}+a_{33} \tau \tau_{1} .
$$

Subtracting Equation 3.9 from Equation 3.8, we get

$$
m_{22} \tau_{2}-a_{22} \tau_{2}+a_{23} \tau_{1}=\tau\left(m_{22} \tau_{1}+a_{32} \tau_{2}-a_{33} \tau_{1}\right)
$$

Looking at Equation 3.6, 3.7 and 3.10, we see that we necessarily have the following three equations

$$
\begin{gathered}
a_{31} \tau_{1}-a_{32}=0, \\
a_{31} \tau_{2}-a_{33}+m_{22}=0, \\
m_{22} \tau_{1}+a_{32} \tau_{2}-a_{33} \tau_{1}=0,
\end{gathered}
$$

otherwise we would have a contradiction with the fact that $\operatorname{Im}(\tau) \neq 0$. Moreover, these last three equations imply also the following three ones

$$
a_{22}-a_{21} \tau_{1}-m_{22}=0,
$$




$$
\begin{gathered}
a_{23}-a_{21} \tau_{2}=0, \\
m_{22} \tau_{2}-a_{22} \tau_{2}+a_{23} \tau_{1}=0 .
\end{gathered}
$$

Now if $a_{31} \neq 0$ and $a_{21} \neq 0$ then we get a contradiction with the fact that $G$ is Cousin by Lemma 5 . Therefore, one of them is necessarily zero. Now, even though the computations are a little bit tedious, the idea is just that in this last case, we shall get a contradiction with the hypothesis that

$$
\mathbb{Z} \subsetneq \operatorname{End}(G) \text {. }
$$

First of all, suppose that both $a_{31}=0$ and $a_{21}=0$, then we get the following equations

$$
a_{31}=a_{21}=a_{32}=a_{23}=0
$$

and

$$
m_{22}=a_{22}=a_{33}
$$

Looking back at the first line of the matrix equation, we get

$$
\begin{gathered}
m_{11}=a_{11}, \\
\left(m_{11}-a_{22}\right) \tau_{1}=a_{12}, \\
\left(m_{11}-a_{33}\right) \tau_{2}=a_{13} .
\end{gathered}
$$

We know that $a_{22}=a_{33}$ and therefore we necessarily have

$$
m_{11}=a_{22}=a_{33}=a_{11},
$$

otherwise, we would get again a contradiction with Lemma 5. Moreover,

$$
a_{12}=a_{13}=0 .
$$

But then, it would mean that every $M(\lambda)$ for all $\lambda \in O$ would be of the form

$$
M(\lambda)=\left(\begin{array}{cc}
n & 0 \\
0 & n
\end{array}\right)
$$

for some integer $n$, and this would be a contradiction with the fact that $\mathbb{Z} \subsetneq \operatorname{End}(G)$.

Hence, we are just left to prove that $a_{31}=0$ implies $a_{21}=0$ and viceversa. Suppose first that $a_{31}=0$, then Equation 3.12 implies

$$
m_{22}=a_{33} \in \mathbb{Z},
$$

but then Equation 3.14 implies

$$
a_{21} \tau_{1}=a_{22}-m_{22},
$$

and Equation 3.15 implies

$$
a_{21} \tau_{2}=a_{23}
$$

This is again a contradiction with Lemma 5 unless $a_{21}=0$.

Finally, if $a_{21}=0$ Equation 3.14 implies

$$
m_{22}=a_{22} \in \mathbb{Z}
$$


but then Equation 3.11 says

$$
a_{31} \tau_{1}=a_{32},
$$

and Equation 3.12 says

$$
a_{31} \tau_{2}=a_{33}-m_{22}
$$

and this is a contradiction unless $a_{31}=0$. This is what we wanted to prove.

Remark. We note that we arrive at the following conclusion. After choosing a lattice representation of $G$, the analytic representation $\rho_{a}$ of $K=\operatorname{End}_{0}(G)$ is isomorphic to a matrix representation of the form

$$
\lambda \mapsto\left(\begin{array}{cc}
m_{11}(\lambda) & 0 \\
m_{21}(\lambda) & m_{22}(\lambda)
\end{array}\right),
$$

where $m_{11}$ is a real embedding of $K$ and $m_{22}$ is a complex embedding of $K$. We shall use this fact in the proof of Lemma 10 .

We are now at the point where we ask: Are there any Cousin groups which have extra multiplication? The answer is yes and we construct such examples over here.

Theorem 9. Let $K$ be a non-totally real cubic number field, and let $\Phi=$ $\left\{\sigma_{1}, \sigma_{2}\right\}$ be a type. Let as usual $\mu_{\Phi}$ be the corresponding Minkowski map and let also $\mathfrak{a}$ be any fractional ideal of $K$, then

$$
\mathbb{C}^{2} / \mu_{\Phi}(\mathfrak{a})
$$

is a Cousin group having extra multiplication. Moreover, for any $\lambda \in O_{K}$ the matrix

$$
\iota(\lambda)=\left(\begin{array}{cc}
\sigma_{1}(\lambda) & 0 \\
0 & \sigma_{2}(\lambda)
\end{array}\right)
$$

induces an endomorphism of $\mathbb{C}^{2} / \mu_{\Phi}(\mathfrak{a})$ which we shall denote by the same symbol $\iota(\lambda)$. The map

$$
O_{K} \rightarrow \operatorname{End}\left(\mathbb{C}^{2} / \mu_{\Phi}(\mathfrak{a})\right),
$$

defined by $\lambda \mapsto \iota(\lambda)$ induces isomorphisms

$$
O_{K} \simeq \operatorname{End}\left(\mathbb{C}^{2} / \mu_{\Phi}(\mathfrak{a})\right)
$$

and

$$
K \simeq \operatorname{End}_{0}\left(\mathbb{C}^{2} / \mu_{\Phi}(\mathfrak{a})\right) .
$$

Proof. It is clear that $\iota(\lambda)$ for any $\lambda \in O_{K}$ induces an endomorphism of $\mathbb{C}^{2} / \mu_{\Phi}(\mathfrak{a})$ because if $\left(\sigma_{1}(\alpha), \sigma_{2}(\alpha)\right) \in \mu_{\Phi}(\mathfrak{a})$ then

$$
\iota(\lambda) \cdot\left(\begin{array}{l}
\sigma_{1}(\alpha) \\
\sigma_{2}(\alpha)
\end{array}\right)=\left(\begin{array}{cc}
\sigma_{1}(\lambda) & 0 \\
0 & \sigma_{2}(\lambda)
\end{array}\right) \cdot\left(\begin{array}{l}
\sigma_{1}(\alpha) \\
\sigma_{2}(\alpha)
\end{array}\right)=\left(\begin{array}{l}
\sigma_{1}(\lambda \cdot \alpha) \\
\sigma_{2}(\lambda \cdot \alpha)
\end{array}\right)
$$


and $\left(\sigma_{1}(\lambda \cdot \alpha), \sigma_{2}(\lambda \cdot \alpha)\right) \in \mu_{\Phi}(\mathfrak{a})$ since $\mathfrak{a}$ is a fractional ideal. This also implies that this Cousin group has extra multiplication. Consider the map

$$
\iota: O_{K} \hookrightarrow \operatorname{End}\left(\mathbb{C}^{2} / \mu_{\Phi}(\mathfrak{a})\right) \text {, defined by } \lambda \mapsto \iota(\lambda) \text {. }
$$

After tensoring with $\mathbb{Q}$ we obtain the map

$$
K \hookrightarrow \operatorname{End}_{0}\left(\mathbb{C}^{2} / \mu_{\Phi}(\mathfrak{a})\right)
$$

which has to be an isomorphism since $\operatorname{End}_{0}\left(\mathbb{C}^{2} / \mu_{\Phi}(\mathfrak{a})\right)$ is a vector space of dimension 3 over $\mathbb{Q}$. We also conclude that $\operatorname{End}\left(\mathbb{C}^{2} / \mu_{\Phi}(\mathfrak{a})\right)$ is an order in $K$, and since it contains $O_{K}$ and $O_{K}$ is the maximal order, we do get that the map $\iota$ in Equation 3.17 is an isomorphism.

Now we can ask if all Cousin groups $G$ of complex dimension 2 and rank 3 having extra multiplication such that $\operatorname{End}(G)$ is the maximal order in a non-totally real cubic number field arise in this way. This is indeed the case, but we begin with a lemma.

Lemma 10. Let $\mathbb{C}^{2} / \Gamma$ be a Cousin group of complex dimension 2 and rank 3. Suppose it has extra multiplication so that

$$
K=\operatorname{End}_{0}\left(\mathbb{C}^{2} / \Gamma\right)
$$

is a non-totally real cubic number field (Theorem 8). Let $\rho_{a}$ be the analytic representation. Then

$$
\rho_{a} \simeq \sigma_{1} \oplus \sigma_{2},
$$

where $\sigma_{1}$ is the unique real embedding of $K$ and $\sigma_{2}$ is one of the two complex embedding of $K$.

Proof. By the remark following Theorem 8, we know that $\rho_{a}$ is isomorphic to a matrix representation $K \rightarrow \mathrm{M}_{2}(\mathbb{C})$ of the form

$$
\lambda \mapsto\left(\begin{array}{cc}
m_{11}(\lambda) & 0 \\
m_{21}(\lambda) & m_{22}(\lambda)
\end{array}\right),
$$

where $m_{11}$ is the real embedding of $K$ and $m_{22}$ is one of the two complex embedding. Since this is a representation, we have that

$$
M\left(\lambda \lambda^{\prime}\right)=M(\lambda) M\left(\lambda^{\prime}\right),
$$

for all $\lambda \in K$. This means the following:

$$
\begin{aligned}
&\left(\begin{array}{cc}
m_{11}(\lambda) & 0 \\
m_{21}(\lambda) & m_{22}(\lambda)
\end{array}\right) \cdot\left(\begin{array}{cc}
m_{11}\left(\lambda^{\prime}\right) & 0 \\
m_{21}\left(\lambda^{\prime}\right) & m_{22}\left(\lambda^{\prime}\right)
\end{array}\right)= \\
&\left(\begin{array}{cc}
m_{11}(\lambda) m_{11}\left(\lambda^{\prime}\right) & 0 \\
m_{21}(\lambda) m_{11}\left(\lambda^{\prime}\right)+m_{22}(\lambda) m_{21}\left(\lambda^{\prime}\right) & m_{22}(\lambda) m_{22}\left(\lambda^{\prime}\right)
\end{array}\right)
\end{aligned}
$$

Therefore, we get

$$
m_{21}\left(\lambda \lambda^{\prime}\right)=m_{21}(\lambda) m_{11}\left(\lambda^{\prime}\right)+m_{22}(\lambda) m_{21}\left(\lambda^{\prime}\right),
$$


and since $K$ is commutative, we have

$$
m_{21}(\lambda) m_{11}\left(\lambda^{\prime}\right)+m_{22}(\lambda) m_{21}\left(\lambda^{\prime}\right)=m_{21}\left(\lambda^{\prime}\right) m_{11}(\lambda)+m_{22}\left(\lambda^{\prime}\right) m_{21}(\lambda),
$$

for all $\lambda, \lambda^{\prime} \in K$. Hence we get

$$
\frac{m_{21}(\lambda)}{m_{11}(\lambda)-m_{22}(\lambda)}=\frac{m_{21}\left(\lambda^{\prime}\right)}{m_{11}\left(\lambda^{\prime}\right)-m_{22}\left(\lambda^{\prime}\right)},
$$

as long as $\lambda, \lambda^{\prime} \notin \mathbb{Q}$. Call this constant $p$, i.e.

$$
p=\frac{m_{21}(\lambda)}{m_{11}(\lambda)-m_{22}(\lambda)},
$$

for all $\lambda \notin \mathbb{Q}$, and consider the matrix

$$
P=\left(\begin{array}{cc}
1 & 0 \\
-p & 1
\end{array}\right)
$$

A simple computation shows that

$$
P \cdot M(\lambda) \cdot P^{-1}=\left(\begin{array}{cc}
m_{11}(\lambda) & 0 \\
0 & m_{22}(\lambda)
\end{array}\right)
$$

for all $\lambda \in K$, and this is exactly what we wanted to show.

Remark. In the classical case of complex tori (the compact case), it is easy to show that $\rho_{r} \simeq \rho_{a} \oplus \overline{\rho_{a}}$. There is thus no difficulty whatsoever to define the notion of type, because it is known that the rational representation is just the sum of the embeddings. More precisely, let $\mathbb{C}^{n} / \Gamma$ be an abelian manifold (meaning a complex torus admitting a Riemann form) having complex multiplication by the $C M$-field $K$ of degree $2 n$ over $\mathbb{Q}$, i.e. we have an embedding

$$
\iota: K \hookrightarrow \operatorname{End}_{0}\left(\mathbb{C}^{n} / \Gamma\right)
$$

We then have associated to these data an analytic and a rational representation. These induce representations of the $C M$-field $K$, and since it is known that

and $\rho_{r} \simeq \rho_{a} \oplus \overline{\rho_{a}}$, we get

$$
\rho_{r} \simeq \bigoplus_{\sigma \in \operatorname{Hom}_{\mathbb{Q}-\text { alg. }}(K, \mathbb{C})} \sigma,
$$

$$
\rho_{a} \simeq \sigma_{1} \oplus \ldots \oplus \sigma_{n}
$$

for some $\sigma_{i}$ where none of them are complex conjugate to each other. This defines then the $C M$-type $\Phi=\left\{\sigma_{1}, \ldots, \sigma_{n}\right\}$, and one says in this case that $\left(\mathbb{C}^{n} / \Gamma, \iota\right)$ is of type $(K, \Phi)$.

Definition 8. Let $G$ be a Cousin group of complex dimension 2 and rank 3 having multiplication by the non-totally real cubic number field $K$, i.e. we have an isomorphism

$$
\iota: K \rightarrow \operatorname{End}_{0}(G)
$$


We know by Lemma 10 that $\rho_{a} \simeq \sigma_{1} \oplus \sigma_{2}$ where $\sigma_{1}$ is the real embedding of $K$ and $\sigma_{2}$ is one of the two complex embedding of $K$. This defines a type $\Phi=\left\{\sigma_{1}, \sigma_{2}\right\}$ of $K$. In this case, we shall say that $(G, \iota)$ is of type $(K, \Phi)$.

Theorem 10. Let $K$ be a non-totally real cubic number field, $\Phi=\left\{\sigma_{1}, \sigma_{2}\right\}$ a type, and let $(G, \iota)$ be a Cousin group (of complex dimension 2 and rank $3)$ of type $(K, \Phi)$. Suppose moreover that

$$
\iota\left(O_{K}\right)=\operatorname{End}(G) .
$$

Then there exists a fractional ideal $\mathfrak{a}$ in $K$ such that

$$
G \simeq \mathbb{C}^{2} / \mu_{\Phi}(\mathfrak{a}) .
$$

Proof. Let $\xi: G \rightarrow \mathbb{C}^{2} / \Gamma$ be a lattice representation. Through the following maps

$$
O_{K} \stackrel{\iota}{\rightarrow} \operatorname{End}(G) \stackrel{\tilde{\xi}}{\rightarrow} \operatorname{End}\left(\mathbb{C}^{2} / \Gamma\right) \stackrel{\rho_{r}}{\rightarrow} \operatorname{End}_{\mathbb{Z}}(\Gamma),
$$

we can consider $\Gamma$ as an $O_{K}$-module. More precisely, if we set $S=\rho_{r} \circ \tilde{\xi}$ the $O_{K}$-action is given by

$$
\lambda \cdot \gamma=S \circ \iota(\lambda)(\gamma)
$$

for all $\lambda \in O_{K}$ and all $\gamma \in \Gamma$.

Let $\gamma_{0} \in \Gamma$ be different from zero and set

$$
\Gamma^{\prime}=O_{K} \cdot \gamma_{0} \subseteq \Gamma .
$$

The map $O_{K} \rightarrow \Gamma^{\prime}$ defined by

$$
\lambda \mapsto \lambda \cdot \gamma_{0},
$$

is an isomorphism of $O_{K}$-module. Hence, we conclude that $\Gamma^{\prime}$ is a free $\mathbb{Z}$-module of rank 3 . There exists then a strictly positive integer $m$ such that

$$
m \Gamma \subseteq \Gamma^{\prime} .
$$

Fix such a $m$, we then get a morphism of $O_{K}$-module

$$
f: \Gamma \rightarrow O_{K} \text {, defined by } \gamma \mapsto f(\gamma),
$$

where $f(\gamma)$ is defined by the equation

$$
m \gamma=f(\gamma) \cdot \gamma_{0}
$$

Let $\mathfrak{a}=f(\Gamma)$, then $\mathfrak{a}$ is an ideal of $O_{K}$. Set $\gamma_{1}=\gamma_{0} / m$, we have

$$
\Gamma=\mathfrak{a} \cdot \gamma_{1}=S \circ \iota(\mathfrak{a})\left(\gamma_{1}\right) .
$$

Since $(G, \iota)$ is of type $(K, \Phi)$, we can find a basis such that the associated matrix representation $M^{\prime}$ is of the form

$$
M^{\prime}(\iota(\lambda))=\left(\begin{array}{cc}
\sigma_{1}(\lambda) & 0 \\
0 & \sigma_{2}(\lambda)
\end{array}\right)
$$


for all $\lambda \in O_{K}$. Let $\left(b_{1}, b_{2}\right)$ be the coordinates of $\gamma_{1}$ with respect to this basis. Then

$$
\Gamma=S \circ \iota(\mathfrak{a})\left(\gamma_{1}\right)=M^{\prime} \circ \iota(\mathfrak{a})\left(b_{1}, b_{2}\right)^{t}=\left\{\left(\sigma_{1}(\alpha) b_{1}, \sigma_{2}(\alpha) b_{2}\right) \mid \alpha \in \mathfrak{a}\right\} .
$$

Now, it is clear that $b_{1} \neq 0$ and $b_{2} \neq 0$, otherwise $\Gamma$ would be contained in either

$$
\mathbb{C} \times\{0\} \text { or }\{0\} \times \mathbb{C},
$$

but this is impossible since $\operatorname{rank}_{\mathbb{Z}}(\Gamma)=3$. After the change of basis

$$
e_{i} \mapsto b_{i}^{-1} e_{i},
$$

we get that that the new matrix representation, say $M$, is still of the form

$$
M(\iota)(\lambda)=\left(\begin{array}{cc}
\sigma_{1}(\lambda) & 0 \\
0 & \sigma_{2}(\lambda)
\end{array}\right)
$$

for all $\lambda \in O_{K}$. Moreover, we get

$$
\Gamma=\mu_{\Phi}(\mathfrak{a}) .
$$

This is what we wanted to prove.

This concludes the study of the ring of endomorphisms of Cousin groups of complex dimension 2 and of rank 3 .

Remark. The notion of types does not appear in the quadratic imaginary case because of the following reason. There are only two types; let $\Phi=\{\sigma\}$ be one of the two types. The other one is then $\bar{\Phi}=\{\bar{\sigma}\}$. Let $\rho$ be the unique non-trivial Galois automorphism of $K$ over $\mathbb{Q}$. In other words, it is the complex conjugation of this $C M$-field. We then have

$$
\mathbb{C} / \mu_{\bar{\Phi}}(\mathfrak{a})=\mathbb{C} / \mu_{\Phi}(\rho(\mathfrak{a})) .
$$

Therefore, one does not miss anything by working only with one of the two $C M$-types. This is typically what one does by viewing a quadratic imaginary number field already inside of $\mathbb{C}$, since this is the same as choosing one of the two embeddings.

In the case of non-totally real cubic number fields, it is not clear to us whether there is a link or not between

$$
\mathbb{C}^{2} / \mu_{\Phi}(\mathfrak{a}) \text { and } \mathbb{C}^{2} / \mu_{\bar{\Phi}}(\mathfrak{a}),
$$

and because of that, the notion of $C M$-types seems to be important. 
3.3. The action of the class group. In this section, we shall work with a fixed non-totally real cubic number field $K$ and a fixed type $\Phi=\left\{\sigma_{1}, \sigma_{2}\right\}$. The gothic letters $\mathfrak{a}, \mathfrak{b}$, and $\mathfrak{c}$ will stand as usual for fractional ideals of $K$. The symbol $\mathrm{Cl}_{K}$ denotes the class group of $K$, and $h_{K}$ denotes its cardinality. It is well-known that $h_{K}$ is finite. Moreover, whenever we say Cousin group, we mean a Cousin group of complex dimension 2 and rank 3. Recall also that given $\mathbb{C}^{2} / \Gamma$, we have the associated matrix representation with respect to the canonical basis of $\mathbb{C}^{2}$ which we denote by

$$
\phi \mapsto M_{\phi}
$$

More generally, if we are given a morphism

$$
\phi: \mathbb{C}^{2} / \Gamma_{1} \rightarrow \mathbb{C}^{2} / \Gamma_{2},
$$

then there is the corresponding lift $L_{\phi}$ and we shall denote the matrix associated to $L_{\phi}$ by $M_{\phi}$. We shall also use the following notation: If $\lambda \in K$ then $M(\lambda)$ will stand for the matrix

$$
\left(\begin{array}{cc}
\sigma_{1}(\lambda) & 0 \\
0 & \sigma_{2}(\lambda)
\end{array}\right) .
$$

Note also that any Cousin group of the form $\mathbb{C}^{2} / \mu_{\Phi}(\mathfrak{a})$ is of type $(K, \Phi)$. Moreover, this property is preserved under isomorphism.

Let then $\Sigma_{\Phi}$ be the set of isomorphism classes of Cousin groups of type $(K, \Phi)$. We shall now explain that there is an action of $\mathrm{Cl}_{K}$ on $\Sigma_{\Phi}$ and it turns out that this action is simply transitive. We use the symbol [] to denote an equivalence class both in $\mathrm{Cl}_{K}$ and in $\Sigma_{\Phi}$. Note also that for any class in $\Sigma_{\Phi}$ there is a representative of the form $\mathbb{C}^{2} / \mu_{\Phi}(\mathfrak{a})$ for some fractional ideal $\mathfrak{a}$.

The action $\mathrm{Cl}_{K} \times \Sigma_{\Phi} \rightarrow \Sigma_{\Phi}$ will be given by

$$
[\mathfrak{a}] \cdot\left[\mathbb{C}^{2} / \mu_{\Phi}(\mathfrak{b})\right]=\left[\mathbb{C}^{2} / \mu_{\Phi}(\mathfrak{a} \mathfrak{b})\right] .
$$

In order to see that this, indeed, induces a simply transitive action, we just need one lemma from which everything else is a consequence.

Lemma 11. Suppose that we have an isomorphism

$$
\phi: \mathbb{C}^{2} / \mu_{\Phi}(\mathfrak{b}) \stackrel{\simeq}{\rightarrow} \mathbb{C}^{2} / \mu_{\Phi}(\mathfrak{c}) .
$$

There exists then $\lambda \in K$ such that

$$
M_{\phi}=M(\lambda) .
$$

Moreover, this $\lambda$ has the property that

$$
\lambda \cdot \mathfrak{b}=\mathfrak{c} .
$$


Proof. By the approximation theorem we can find $\lambda_{1} \in K^{\times}$such that $\lambda_{1} \mathfrak{c} \subseteq \mathfrak{b}$. The matrix $M\left(\lambda_{1}\right)$ induces a morphism

$$
\mathbb{C}^{2} / \mu_{\Phi}(\mathfrak{c}) \rightarrow \mathbb{C}^{2} / \mu_{\Phi}(\mathfrak{b}),
$$

which we denote by the same symbol $M\left(\lambda_{1}\right)$. Consider then the endomorphism $M\left(\lambda_{1}\right) \circ \phi$ of $\mathbb{C}^{2} / \mu_{\Phi}(\mathfrak{b})$ :

$$
\mathbb{C}^{2} / \mu_{\Phi}(\mathfrak{b}) \stackrel{\phi}{\longrightarrow} \mathbb{C}^{2} / \mu_{\Phi}(\mathfrak{c}) \stackrel{M\left(\lambda_{1}\right)}{\longrightarrow} \mathbb{C}^{2} / \mu_{\Phi}(\mathfrak{b}) .
$$

Its lift in matrix form is given by $M\left(\lambda_{2}\right)$ for some $\lambda_{2} \in K^{\times}$. Note that both $M\left(\lambda_{1}\right)$ and $M\left(\lambda_{2}\right)$ are non-singular. We then have

$$
M\left(\lambda_{1}\right) \cdot M_{\phi}=M\left(\lambda_{2}\right) .
$$

Since $M\left(\lambda_{1}\right)$ is non-singular, we get

$$
M_{\phi}=M(\lambda)
$$

where $\lambda=\lambda_{1}^{-1} \lambda_{2}$.

Now, we clearly have $\lambda \cdot \mathfrak{b} \subseteq \mathfrak{c}$. Since $\lambda_{1} \cdot \mathfrak{c} \subseteq \mathfrak{b}$, we see that $\lambda_{2}^{-1} \lambda_{1} \cdot \mathfrak{c} \subseteq \mathfrak{b}$, and we conclude that $\lambda \cdot \mathfrak{b}=\mathfrak{c}$.

Proposition 1. Suppose we have an isomorphism

$$
\phi: \mathbb{C}^{2} / \mu_{\Phi}(\mathfrak{b}) \stackrel{\simeq}{\rightarrow} \mathbb{C}^{2} / \mu_{\Phi}(\mathfrak{c}),
$$

and suppose that $\mathfrak{a}$ is any fractional ideal of $K$. We then have

$$
\mathbb{C}^{2} / \mu_{\Phi}(\mathfrak{a} \mathfrak{b}) \simeq \mathbb{C}^{2} / \mu_{\Phi}(\mathfrak{a c}) .
$$

Proof. By Lemma 11, we know that $M_{\phi}=M(\lambda)$ for some $\lambda \in K^{\times}$satisfying $\lambda \cdot \mathfrak{b}=\mathfrak{c}$. This induces an isomorphism

$$
\mathbb{C}^{2} / \mu_{\Phi}(\mathfrak{a} \mathfrak{b}) \simeq \mathbb{C}^{2} / \mu_{\Phi}(\mathfrak{a} \mathfrak{c})
$$

its inverse being induced by $M\left(\lambda^{-1}\right)$.

Proposition 2. Let $\mathfrak{a}, \mathfrak{a}^{\prime}$ be fractional ideals of $K$ and suppose that $[\mathfrak{a}]=$ $\left[\mathfrak{a}^{\prime}\right]$. Suppose also that we are given $\mathbb{C}^{2} / \mu_{\Phi}(\mathfrak{b})$. We then have

$$
\mathbb{C}^{2} / \mu_{\Phi}(\mathfrak{a} \mathfrak{b}) \simeq \mathbb{C}^{2} / \mu_{\Phi}\left(\mathfrak{a}^{\prime} \mathfrak{b}\right)
$$

Proof. Since $[\mathfrak{a}]=\left[\mathfrak{a}^{\prime}\right]$, there exists $\lambda \in K^{\times}$such that $\lambda \mathfrak{a}=\mathfrak{a}^{\prime}$. Consider $M(\lambda)$, similarly as in the proof of Proposition 1, it induces an isomorphism

$$
\mathbb{C}^{2} / \mu_{\Phi}(\mathfrak{a} \mathfrak{b}) \simeq \mathbb{C}^{2} / \mu_{\Phi}\left(\mathfrak{a}^{\prime} \mathfrak{b}\right)
$$

Remark. Propositions 1 and 2 prove in fact that the action is well-defined.

Proposition 3. The action above is simply transitive. 
Proof. The fact that it is transitive follows from the following remark. Given any two Cousin groups of the form

$$
\mathbb{C}^{2} / \mu_{\Phi}(\mathfrak{b}) \text { and } \mathbb{C}^{2} / \mu_{\Phi}(\mathfrak{c})
$$

we have

$$
\left[\mathfrak{b}^{-1} \mathfrak{c}\right] \cdot\left[\mathbb{C}^{2} / \mu_{\Phi}(\mathfrak{b})\right]=\left[\mathbb{C}^{2} / \mu_{\Phi}(\mathfrak{c})\right]
$$

Similarly, the simple transitiveness follows from the fact that

$$
\mathbb{C}^{2} / \mu_{\Phi}(\mathfrak{a} \mathfrak{b}) \simeq \mathbb{C}^{2} / \mu_{\Phi}\left(\mathfrak{a}^{\prime} \mathfrak{b}\right)
$$

implies that there exist $\lambda \in K^{\times}$such that

$$
\lambda \mathfrak{a} \mathfrak{b}=\mathfrak{a}^{\prime} \mathfrak{b},
$$

by Lemma 11. But then multiplying by $\mathfrak{b}^{-1}$, we get

$$
\lambda \mathfrak{a}=\mathfrak{a}^{\prime},
$$

that is $[\mathfrak{a}]=\left[\mathfrak{a}^{\prime}\right]$.

Corollary 7. There are exactly $h_{K}$ isomorphism classes of Cousin groups of type $(K, \Phi)$.

Proof. This follows immediately from Proposition 3.

We thus see that the arithmetic of non-totally real cubic number fields is intimately related with Cousin groups of complex dimension 2 and rank 3 having extra multiplication. As we said in the introduction, we postpone the study of meromorphic functions on the connected abelian complex Lie groups $\mathbb{C}^{2} / \mu_{\Phi}(\mathfrak{a})$ to a further paper.

\section{References}

[1] F. Capocasa and F. Catanese, Periodic meromorphic functions. Acta Math. 166 (1991), 1-2, 27-68.

[2] P. Cousin, Sur les fonctions triplement périodiques de deux variables. Acta. Math. 33 (1910), 1, 105-232.

[3] F. Gherardelli, Varieta' quasi abeliane a moltiplicazione complessa. Rend. Sem. Mat. Fis. Milano. 57 (1989), 8, 31-36.

[4] P. DE LA HARPE, Introduction to complex tori. Complex analysis and its applications (Lectures, Internat. Sem., Trieste, 1975), Vol. II, pp. 101-144. Internat. Atomic Energy Agency, Vienna, 1976.

[5] A. Morimoto, On the classification of noncompact complex abelian Lie groups. Trans. Amer. Math. Soc. 123 (1966), 220-228.

[6] G. Shimura, Introduction to the arithmetic theory of automorphic functions. Princeton University Press, 1994.

[7] G. Shimura and Y. Taniyama, Complex multiplication of abelian varieties and its applications to number theory. The Mathematical Society of Japan, 1961.

[8] G. Shimura, Abelian varieties with complex multiplication and modular functions. Princeton University Press, 1998.

[9] C. L. Siegel, Topics in complex function theory I, II, III. Wiley-Interscience, 1969. 
Daniel VALLIÈRES

University of California, San Diego

9500 Gilman Drive \# 0112

La Jolla, CA 92093-0112

USA

Current address :

Fakultät für Mathematik

Institut für theoritische Informatik und Mathematik

Universität der Bundeswehr Münschen

85577 Neubiberg

Deutschland

E-mail: dvallieres@math.ucsd.edu

$U R L:$ http://www. math.ucsd.edu/ dvallier 